\title{
Lumen
}

Selected Proceedings from the Canadian Society for Eighteenth-Century Studies

\section{Figures du recueil : les contes de fées en leurs Cabinets au XVIII $^{\mathrm{e}}$ siècle}

\section{Aurélie Zygel-Basso et Amélia Belin}

Volume 29, 2010

URI : https://id.erudit.org/iderudit/1012027ar

DOI : https://doi.org/10.7202/1012027ar

Aller au sommaire du numéro

Éditeur(s)

Canadian Society for Eighteenth-Century Studies / Société canadienne d'étude du dix-huitième siècle

ISSN

1209-3696 (imprimé)

1927-8284 (numérique)

Découvrir la revue

Citer cet article

Zygel-Basso, A. \& Belin, A. (2010). Figures du recueil : les contes de fées en leurs Cabinets au XVIII ${ }^{\mathrm{e}}$ siècle. Lumen, 29, 71-89. https://doi.org/10.7202/1012027ar

Copyright @ Canadian Society for Eighteenth-Century Studies / Sociéte canadienne d'étude du dix-huitième siècle, 2010
Ce document est protégé par la loi sur le droit d'auteur. L'utilisation des services d'Érudit (y compris la reproduction) est assujettie à sa politique d'utilisation que vous pouvez consulter en ligne.

https://apropos.erudit.org/fr/usagers/politique-dutilisation/ 


\section{Figures du recueil : les contes de fées en leurs Cabinets au XVIIIe siècle}

On commencera par un paradoxe : l'autonomie des contes merveilleux, peu commune vers 1700 , est de nos jours une condition nécessaire à leur diffusion. Jean-Paul Sermain a montré comment les principales anthologies grand public de contes des XVII ${ }^{\mathrm{e}}$ et $\mathrm{XVIII}$ siècles, ces quinze dernières années, avaient choisi d'éliminer toutes les formes de cadres. $C^{\prime}$ est un phénomène étrange que cette survie des textes hors des récits dans lesquels ils étaient insérés et qui les liaient : «L'oubli du recueil, du cadre, de l'insertion sont la contrepartie d'une propriété inhérente au conte, sa circulation et la quasi autonomie de l'histoire qu'il raconte, et en même temps, pourtant, le recueil, le cadre et l'insertion sont les conditions mêmes de cette liberté. [...] À travers l'entourage du conte se lit sa participation à une histoire [...] de longue durée, dessinant les lignes généalogiques de contes remarquables et le devenir d'un genre ${ }^{1} . »$ Le recueil est lui-même un phénomène complexe, tardif et largement consacré à la fin du XVIII siècle. Car au moment où les Cabinets des fées se constituent, le discours sur ce nouveau genre donne à lire une double ambition d'encyclopédie et de stratégie éditoriale, et plus seulement de récolement. Dès les années 1700 , le recueil de contes naît en même temps que se développe une expérience générique nouvelle. Le conte se dit et constitue sa propre mémoire sur plusieurs plans : dans des collections, dans le motif anthologique qui le parcourt, dans ses illustrations, enfin. On verra alors les anthologies de contes de fées formuler sur le genre une doxa qui coïncide avec le tournant moral et pédagogique qu'il prend.

1 Jean-Paul Sermain, "La face cachée du conte, le recueil et l'encadrement», dans Féeries, 1, 2003, p. 14. 


\section{Fictions compilatrices}

Ce que Jean-François Perrin appelle «incrustations anthologiques du genre dans la diégèse du conte ${ }^{2} »$ était présent dès La Chatte blanche de $\mathrm{M}^{\mathrm{me}} \mathrm{d}$ 'Aulnoy, où le héros pouvait lire une histoire de la féerie sur les murs ensorcelés du château. Ces inscrustations, internes à la première vague des conteurs, représentaient un genre en train de naître en même temps qu'elles renvoyaient à la "généalogie féerique ${ }^{3}$ ", soit Aulnoy elle-même, Perrault, $\mathrm{M}^{\text {lle }}$ Lhéritier. Les références désignaient un "archi-recueil de tout récit merveilleux possible postérieurement ${ }^{4}$ ", qui allait de pair avec les traditions romanesque et épique. Précisément, la tradition médiévale et antique de réflexivité, traitée avec humour dans les années 1690, devient fantasme du grand livre vers $1740 \mathrm{chez}$ $\mathrm{M}^{\mathrm{me}}$ de Villeneuve : la scène de lecture par une fée de son grimoire $\mathrm{y}$ est omniprésente. Boîtes secrètes, vélins indéchiffrables, cassettes aux verrous chantournés, grimoires féeriques mis en abyme ne sont plus qu'un accessoire, qui semble cependant obséder les conteuses tardives. Le motif reflète le souci compilatoire de l'époque. L'insistance d'une conteuse comme $\mathrm{M}^{\mathrm{me}}$ de Lintot sur les objets gravés d'inscriptions plus ou moins mystérieuses place ses textes dans un rapport affiché d'intertextualité savante et malicieuse, tant avec les références que sont devenus les textes des conteurs précédents qu'avec la vogue des contes gazés à la Crébillon. Tendrebrun, lorsqu'il affronte la lubrique fée Vicieuse dans Tendrebrun et Constance de Lintot (1735), la voit s'offrir à lui le livre à la main : «Dans une de ses mains était un livre de vélin doré, et dans l'autre une baguette avec laquelle elle lui donna un coup sur l'épaule ${ }^{5} . »$ Et pourtant, la présence du Livre est tournée en dérision, puisque le prince n'est préoccupé que de la croissance accélérée de sa bien-aimée Constance, cachée sous la robe de Vicieuse par un détour arbitraire du conte : «il avait les yeux attachés sur sa robe de chambre, parce qu'il s'apercevait qu'elle remuait, et qu'il ne doutait pas que ce ne

2 Jean-François Perrin, «Recueillir et transmettre, L'effet anthologique dans le conte merveilleux (XVII - -XVIII ${ }^{\mathrm{e}}$ siècle)", dans Féeries, 1, 2003, p. 147.

3 Ibid., p. 148.

4 Ibid.

$5 \quad \mathrm{M}^{\mathrm{me}}$ de Lintot, Tendrebrun et Constance, dans $\mathrm{M}^{\mathrm{me}}$ Levesque, $\mathrm{M}^{\mathrm{me}}$ de Gomez, $\mathrm{M}^{\mathrm{me}}$ de Dreuillet... [et al.], Contes, édition établie par Raymonde Robert, Paris, H. Champion, coll. «Sources classiques», Bibliothèque des Génies et des Fées (BGF), 13, 2007, p. 637. 
fût la malheureuse Constance qui croissait à vue d'œil ${ }^{6} » .$. Ces incrustations anthologiques sont aussi le reflet d'une stratégie éditoriale florissante. Les éditeurs des années 1730 insistent sur le caractère nouveau des recueils et sur leur dimension féerique, comme pour les Nouveaux Contes de fées de $\mathrm{M}^{\mathrm{me}}$ de Lassay (1737), contes à l'édition desquels elle ne semble pas avoir participé. Une autre conteuse, $\mathrm{M}^{\mathrm{me}}$ de Dreuillet, est morte depuis cinq ans lorsque paraît Le Phénix. Raymonde Robert remarque, à propos des contes de $\mathrm{M}^{\text {mes }}$ Dreuillet et Le Marchand :

Intitulée Nouveaux Contes des fées allégoriques, l'édition de 1735 est présentée comme l'oeuvre $d^{\prime}$ un seul individu très imprécis : $M^{* * *}$. Le contenu du recueil est annoncé en ces termes : "Le Phénix, Lisandre, Carline, Boca, etc. ", cette liste étant remarquablement approximative : en effet, Lisandre et Carline ne correspondent qu'à un seul texte et les " etc. " ne renvoient à rien. Il s'agit là, vraisemblablement, d'une stratégie éditoriale qui vise à " gonfler » le recueil en postulant qu'il contient beaucoup plus de récits qu'en réalité. En fait, le contenu réel se limite à trois textes, Le Phénix, Lisandre et Carline et Boca. Chacun est attribué à des personnes différentes, désignées, comme c'est souvent le cas à l'époque, par des lettres que les lecteurs du temps pouvaient probablement décrypter facilement. [...] Le titre général de "contes de fées» contient une part de vrai, mais une part aussi importante de faux. Deux récits seulement correspondent, en effet, à ce qui est annoncé : "Le Phénix ", et l' "Histoire de la princesse Abdelasis", récit inséré dans Boca. Il s'agit là effectivement de contes de fées dans la plus pure tradition du genre ${ }^{7}$.

Cette nouveauté revendiquée n'est donc pas liée à une originalité qu'afficherait l'auteur dans son rapport au genre du conte de fées : l'édition fonctionne par référence aux grands conteurs et conteuses de la première vague (vers 1690). Comme pour la poétique du conte, les stratégies éditoriales des années 1730 s'appuient sur les attentes des lecteurs et sur la continuation d'une lignée de prédécesseurs célèbres. Et si les contes licencieux sont publiés de façon autonome après 1730, cela s'explique sans doute par leur taille proche de celle des romans. On voit alors se dessiner une démarche différente de ce qui s'est pratiqué jusque-là : le premier Cabinet des fées paraît en $1717^{8}$. Puis en 1731

6 Ibid.

$7 \mathrm{M}^{\mathrm{me}}$ Levesque, $\mathrm{M}^{\mathrm{me}}$ de Gomez, $\mathrm{M}^{\mathrm{me}}$ de Dreuillet... [et al.], op. cit., p. 463.

8 Le Cabinet des fées, contenant les contes de fées, Amsterdam, 1717, 6 vol. 
et 1754 , à différentes adresses, sont publiés Les Cabinets des fées ${ }^{9}$, et en 1748 la Bibliothèque choisie et amusante ${ }^{10}$, collection de contes (on en trouvera aussi beaucoup dans la Bibliothèque universelle des romans ${ }^{11}$, dans les Lectures sérieuses et amusantes ${ }^{12}$ ), mais on est encore loin de la somme de $1785-1789^{13}$. Pour cette sélection de Mayer, le Cabinet des fées ou collection choisie des contes des fées et autres contes merveilleux, qui englobe $L a$ Bibliothèque des génies et des fées de $1765^{14}$, on voit à quel point le regard a changé, avec ce prospectus qui avance à propos des contes de fées que «leur collection ne peut manquer d'être accueillie, n'eût-elle encore que le mérite de réunir des membres épars, qu'il est impossible de trouver, \& de reparoître à nos yeux comme une galerie de tableaux des meilleurs maîtres anciens, dont on fait revivre les couleurs ${ }^{15}$.»

On notera l'importance de l'image du tableau dans cette deuxième moitié de siècle qui vit avec les Salons ${ }^{16}$. La préface de la Nouvelle Bibliothèque Bleue par Jean Castilhon brode sur le motif à propos des textes du Moyen-Âge : «Outre qu'ils ne sont presque tous que des traductions informes, ils sont écrits d'une manière si barbare, que vous auriez de la

9 Les Cabinets des fées, contenant leurs ouvrages en huit volumes, Amsterdam, Michel Charles Le Cene, 1731-1735, et Les Cabinets des fées, contenant leurs ouvrages en huit volumes, Amsterdam, M.-M. Rey, 1754-1761 [8 vol. in-12, vol. 9 en 1735, nouvelle édition par $\mathrm{M}^{\mathrm{me}}$ de $\mathrm{M}^{* \cdots}$ en 1754 , même éditeur, même adresse].

10 Bibliothèque choisie et amusante, Amsterdam, aux dépens de la Compagnie, 17481752,6 tomes en 3 vol.

11 Bibliothèque universelle des romans, Paris, Lacombe et Pancoucke, 112 vol. in-12, et Slatkine reprints, Genève, 1970, voir Table analytique générale, 13 p.

12 Lectures sérieuses et amusantes, Genève, Antoine Philibert, 1753, 6 vol. in-12.

13 Voir aussi les Voyages imaginaires, romanesques, merveilleux, allégoriques, amusans, comiques et critiques, suivis de songes et visions et des romans cabalistiques, Amsterdam et Paris, Hôtel Serpente, 1787-1789, 36 vol. in-12. Notre édition complète des dessins préparatoires du Cabinet des fées et des Voyages imaginaires paraîtra aux Presses de l'Université Laval en 2012.

14 Bibliothèque des génies et des fées, Paris, Duchesne, 1765, 2 vol. in-12.

15 Le Cabinet des fées ou Collection choisie des contes des fées et autres contes merveilleux ornés de figures, Le, Amsterdam et Paris, rue et hôtel Serpente, 1785-1786 (pour les 37 premiers volumes), Genève, Barde, Manget et Cie / Cuchet, 1788-1789 (pour les 4 derniers), 41 vol. in- $8^{\circ}$, et Le Cabinet des fées ou collection choisie des contes des fées, et autres contes merveilleux..., Paris, Impr. Chardon, 1785, 4 p. Saisie du texte et relecture : O. Bogros pour la collection électronique de la Médiathèque André Malraux de Lisieux (15.I.2007). URL : <http://www.bmlisieux.com/litterature/bibliogr/ cabfees.htm>.

16 Pierre Frantz, L'esthétique du tableau dans le théâtre du XVIII siècle, Paris, PUF, 1998. 
peine à les entendre; ce sont de vieux tableaux qu'il faut raccommoder après les avoir lavés, et à plusieurs desquels il faut mettre des fonds ${ }^{17}$.» Certes, dit le compilateur dialoguant avec une dame, "Quant à cette imagination que vous trouvez si brillante, il n'y a pas un de ces anciens Romans qu'on ne lit plus, qui n'en offre mille fois davantage ${ }^{18} .{ }^{\prime \prime}$ Ailleurs, l'Avertissement de l'édition de 1781 des Mémoires sur l'ancienne chevalerie de La Curne de Sainte-Palaye, avertissement rédigé par JeanPierre de Bougainville, compare la réaction des lecteurs devant des textes médiévaux à l'impression d'étrangeté qui saisit le spectateur de vieux portraits de famille : «La gothicité du costume, l'habillement bizarre, le maintien raide et empesé des personnages qu'ils représentent, nous paraissent tout-à-fait plaisants[...]. Cependant[...] un esprit attentif retrouve[...] encore aujourd'hui dans notre nation tout le caractère des anciens Francs, mélangé de quelques nuances de celui des Gaulois ${ }^{19}$." Mais chez l'éditeur du Cabinet des fées, la comparaison picturale vient soutenir une vision patrimoniale positive développée dans ses notices et dans son prospectus.

\section{Fonder une tradition : le discours sur les conteuses}

L'étude des notices biographiques au volume 37 du Cabinet des fées montre une différence frappante entre celles des conteuses du premier $\mathrm{XVIII}^{\mathrm{e}}$ siècle, encore rattachées à la tradition salonnière, et celle consacrée à $\mathrm{M}^{\text {me }}$ Leprince de Beaumont. Pour cette dernière, un laconisme qui frise le cocasse : «BEAUMONT Le Prince de. Elle est née à Rouen, le 26 Avril 1711. Son mérite, universellement reconnu pour l'éducation, l'a fixée à Londres, environ en 1769. Elle donnoit en 1769, un Journal de morale qui est estimé. Nous croyons qu'elle est morte ${ }^{20} . »$ Le rédacteur est bien plus prolixe, en revanche, lorsqu'il s'agit de Levesque, Lemarchand, Lintot, Lubert et Lussan, sans doute aussi parce qu'il peut puiser dans des notices de dictionnaire déjà existantes. Selon la notoriété plus ou moins grande des conteuses, il les range en deux catégories : celles

17 Jean Castilhon, Nouvelle Bibliothèque bleue, ou Recueil d'histoires singulières et naïves, Paris, Lacombe, 1769, p. xi.

18 Ibid.

19 Jean Baptiste La Curne de Sainte-Palaye, Mémoires sur l'ancienne chevalerie, Paris, Duchesne, 1781, vol. 3, p. [v]-vi. 
qui ont brillé dans la meilleure société, et celles qui sont restées dans une ombre qu'il veut croire choisie. Il se garde bien de préciser (peutêtre l'ignore-t-il, ou se fie-t-il aux épîtres de Voltaire?) que Lubert est restée vieille fille faute de dot, que son amant, le président Roujault, l'a quittée pour la richissime marquise d'Ambre ${ }^{21}$, et que la solitude de Lintot était sans doute du même genre. Quant à la bonne société, encore est-elle limitée aux cercles parlementaires où ont évolué Lemarchand, Levesque (la femme d'un "gendarme vif et enjoué») et Lubert. La notice consacrée à $\mathrm{M}^{\mathrm{lle}}$ de Lussan, aventurière, est pourtant la plus savoureuse et la plus acerbe. L'auteure des Veillées de Thessalie, soupçonnée de plagiat, doit se faire pardonner d'avoir vécu de sa plume et d'avoir donné dans le roman, ce genre «analogue à son caractère \& à ses penchants; car elle étoit laide, sensible \& gourmande; on va loin avec ces trois qualités ${ }^{22} »$ ! L'archétype de la femme de lettres est croqué en quelques traits : qu'elle soit lancée dans le monde ou ensevelie dans la retraite, elle reste avant tout modeste, enjouée, tranquille, et le plus grand mérite de son style est d'être «agréable», «moral», «charmant», «naïf» ou encore "facile». Les conteuses s'inscrivent dans la perspective plus large d'une contribution à l'histoire littéraire des femmes, dont l'éloquence est alors associée à un art de plaire, et dont le rôle dans la République des Lettres est sans cesse associé à cette promotion d'une rhétorique du delectare, développée naturellement et sans avoir, ou presque, appris les artifices d'un métier. La définition du conte est étroitement liée à cette vision des conteuses, et elle s'étend même à tout le merveilleux, si l'on en croit le propos du prospectus.

\section{Le prospectus : définitions et stratégie éditoriale nouvelle}

Mayer fait la différence entre le "poème» qui élève et le «conte» qui charme. On voit se constituer un discours «officiel» sur l'évolution du genre, en quatre points :

1) Le conte des fées est rattaché exclusivement à ses origines orientales.

21 Voir à ce sujet l'introduction de $\mathrm{M}^{\text {Ile }}$ de Lubert, Contes, Aurélie Zygel-Basso (éd.), Paris, H. Champion, coll. "Sources classiques», Bibliothèques des Génies et des Fées, $14,2005$.

22 Cabinet des fées, op. cit., p. 153-154. 
2) Le conte de fées est fédérateur et transcende les groupes sociaux, il se lit en famille.

3) La puissance de l'imagination est célébrée surtout comme un appeau pour l'instruction des enfants et l'édification des adultes, et se retrouve opposée à des systèmes d'éducation plus austères avec la caution d'une citation de l'abbé Lenglet Dufresnoy. Mais on n'ira pas jusqu'à inclure dans le florilège les œuvres des conteurs masculins comme Voltaire, Crébillon, Voisenon, Diderot, qui «en ont écarté la simplicité \& la décence, caractères distinctifs \& recommandables de la Féerie; ce qui prouve seulement qu'ils ont fait un abus de leur génie, abus qui les exclut nécessairement de la Collection que nous offrons au Public, dans laquelle nous ne ferons entrer que des Contes choisis \& propres à remplir les objets indiqués dans ce Prospectus ${ }^{23}$.» Rousseau, mystérieusement, trouve seul grâce pour sa "décence» aux yeux du compilateur, ce qui ne manque pas de provoquer notre surprise quand on songe à la fin de la Reine Fantasque de 1754, où la reine meurt d'une indigestion de ragoût de pieds de perdrix avant de pouvoir coucher avec le roi comme elle l'y avait engagé par ses agaceries... Mayer lui préfère le conte à vraisemblance morale à la Marmontel.

4) Le discours sur le plaisir, qui pouvait aller jusqu'à la provocation dans les préfaces des années 1730, est ici bien lénifiant, puisque ce dernier, cachant quelque vérité, a presque toujours une valeur seconde. Le changement est lié à l'idée nationale du lecteur que véhicule le prospectus; sa conception élargie du public procède aussi, bien sûr, des enjeux économiques qu'impose un lancement de cette envergure.

\section{Les destinataires : acheteurs, lecteurs}

À une époque où le livre reste un objet de luxe, de qualité, qui mise sur l'élégance et dont la diffusion est assez restreinte, Mayer prend le risque de publier la collection du Cabinet des fées, dont l'enjeu était considérable de par son ampleur (vingt-cinq volumes au départ). Il met en place une politique commerciale destinée à viser un lectorat le plus large possible. Pour cela, il s'appuie sur la partie du prospectus qui 
présente la future collection (format, nombre de volumes, présence d'illustrations, prix), et sur la souscription qui permet d'assurer la vente de la collection complète. Celle-ci reste ouverte pendant toute la durée de la parution, et, fait exceptionnel, les souscripteurs n'avancent pas $\mathrm{d}$ 'argent. Le chevalier Mayer fait appel au libraire Cuchet, réputé pour son sérieux (il vient de terminer, en 1785, l'édition in- $8^{\circ}$ des $C E u v r e s$ choisies de Le Sage et de l'Abbé Prévost en quinze et trente-neuf volumes). Pour l'édition princeps, il emploie Clément-Pierre Marillier, dessinateur de renom, dont le sérieux et la réputation ne sont plus à prouver ${ }^{24}$, et le graveur Nicolas de Launay, deux artistes en étroite collaboration.

La première édition du Cabinet des fées est destinée à un public aisé de savants et de collectionneurs puisqu'elle paraît dans un format in $-8^{\circ}$, format des grandes collections, et les planches sont vendues avec, pour la somme de trois livres douze sols, ce qui représente pour la collection en 31 volumes ${ }^{25}$ la somme de cent onze livres six sols, à laquelle il faut rajouter le prix de la reliure ${ }^{26}$. Mayer décide très rapidement d'une seconde édition, de format in-12 - format plus petit, plus pratique et moins cher - destinée à un public plus modeste puisqu'il met en place deux «formules». Pour attirer de nouveaux lecteurs, il vante dans le prospectus le succès de la première édition. Il offre la possibilité à l'acheteur de prendre la collection avec ou sans les planches. Celles-ci sont le plus souvent de moins bonne qualité puisqu'il s'agit de tirages supplémentaires. Les prix sont bien sûr plus abordables (un ouvrage sans planches coûte une livre quinze sols, et avec planches deux livres huit sols), et la collection revient moitié moins cher que la première édition.

24 Marillier venait de terminer en 1785 , les 30 et 77 planches hors-textes des CEuvres choisies de Le Sage et de l'Abbé Prévost. Il collabora de nouveau avec Cuchet pour les Voyages imaginaires où il fournit 77 planches.

25 En juin 1786, paraît un avis dans différents journaux de l'époque où il est annoncé que la collection comporte un supplément de six volumes, que le souscripteur n'est pas obligé de prendre.

26 Les volumes étaient vendus brochés avec les planches séparées. L'acheteur devait donc faire relier chaque volume en y incluant les planches, ce qui explique les éventuelles erreurs de disposition de ces dernières et la somptuosité des reliures suivant l'aisance du lecteur. 


\section{Marillier et les dessins du Cabinet des fées}

De 1750 à 1775, en pleine floraison de ce que les Goncourt appelleront le «siècle de la vignette», l'agrément des figures est parfois un argument de vente à part entière ${ }^{27}$. Les illustrations du Cabinet des fées s'inscrivent dans cette production du livre de semi-luxe, et notre étude laisse ici de côté la Bibliothèque bleue pour tenter de voir comment les figures de Marillier soutiennent le propos anthologique, unique par son ambition, du chevalier de Mayer, en particulier pour la production disparate et inclassable des dernières conteuses. Le Cabinet des fées n'est pas la première anthologie illustrée par Marillier, et il entreprend souvent ses frontispices de la même manière. La disposition avec médaillons et éléments métonymiques au centre, soit par la mention d'un récit particulier, soit par des objets emblématiques, est le fruit d'une mode dans le livre illustrée ${ }^{28}$. Elle correspond graphiquement au passage d'une lecture «intensive» à une lecture «extensive ${ }^{29} »:$ :[Du Moyen-Âge à 1750 environ] les hommes [...] ne disposaient que de quelques livres[...] et ils les relisaient sans cesse, généralement à haute voix et en groupe, de sorte qu'une sélection limitée de littérature traditionnelle se gravait dans leur conscience. Vers $1800,[\ldots]$ ils sont submergés de nouvelles publications $[. .$.$] et ils les dévorent en écartant l'un dès qu'ils peuvent en$

27 Alain-Marie Bassy, «Le texte et l'image» (Histoire de l'édition française, Paris, 1984, $\mathrm{t}$. 2, p. 140-161), et Philip Stewart, Engraven Desire, Eros, Image and Text in the French Eighteenth Century (Duke University Press, Durham-Londres, 1992). Plus récemment, voir l'anthologie de Christophe Martin, "Dangereux suppléments», L'illustration du roman en France au dix-huitième siècle, Louvain-Paris, Peeters, 2005, Nathalie Ferrand, Livres vus, livres lus : une traversée du roman illustré des Lumières, Oxford, Voltaire Foundation, SVEC, 3, 2009, et la thèse de Benoit Tane, intitulée Avec figures..., à paraître aux Presses de l'Université Laval.

28 Boccace, Nouvelles, traduction libre, ornée de la vie de Boccace, des contes que La Fontaine a empruntés de cet auteur et des figures gravées sous la direction de Ponce d'après les dessins de Marillier, par Mirabeau, de l'imprimerie d'Egron, Paris, chez L. Duprat, Letellier et $C^{i e}, 1802,4$ vol. in $-8^{\circ}$. On y trouve huit figures de Marillier entourées d'un cadre de médaillons de formes variées résumant les contes non visés par la figure du milieu.

29 Rolf Engelsing, «Die perioden der Lesergeschichte in der Neuzeit : das statistische Ausmass und die soziokulturelle Bedeutung der Lektüre», Archiv für Geschichte des Buchwesens, 10, 1969. Col. 944-1002. Engelsing, Der Bürger als Leser : Lesergeschichte in Deutschland 1500-1800, Stuttgart, 1974, et David Hall, "The Uses of Literacy in New England 1600-1850", in William L. Joyce, David D. Hall, Richard D. Brown, John B. Hench, Printing and Society in Early America, Worcester, American Antiquarian Society, 1983. Voir Robert Darnton, Gens de lettres, gens du livre, éd. Odile Jacob, Paris [trad. française], 1992, p. 200-202. 
trouver un autre ${ }^{30} . » A$ moment où l'on relit les contes merveilleux du XVIII ${ }^{\mathrm{e}}$ siècle, et avec eux sa production romanesque, l'illustration a tendance à suivre cet éclatement que le public connaît souvent, au moins pour le lectorat amateur et acheteur de recueils de luxe comme celui qui nous occupe. Éclatement de la disposition, éclatement des sources vont de pair avec l'atmosphère troubadour. Les différentes inspirations sont réparties selon les textes choisis par Mayer pour le Cabinet des fées, elles servent aussi son propos d'ensemble.

Le choix des titres donnés aux figures est, bien sûr, chargé de sens. Il n'est que de comparer l'intitulé des planches, au fur et à mesure que l'on avance dans les volumes, pour le voir. Cinq atmosphères se dégagent des illustrations de Marillier : la veine folklorique, la tonalité galante et pastorale, l'exotisme orientalisant, la malice parodique, enfin l'air de tragédie lyrique. Or, que nous disent Mayer, Marillier et leurs graveurs? Chaque registre appartient à une production définie, à un type de conte. Un lecteur attentif sait bien qu'il n'en est rien; les contes d'Aulnoy savent mêler gourmandise et bergeries, les contes orientaux voyagent du côté de la satire, mais aussi du roman d'aventures, les contes libertins regorgent de développements sentimentaux dont l'analyse est souvent très fine. Mais ici, chacun est prié de rester dans son domaine. Cette réception inscrite à l'intérieur du XVIII siècle nous montre à quel point le propos des libraires a changé.

Qu'on en juge par un balayage, même rapide, de ces titres. Si l'on se limite aux conteuses, on note une différence de ton flagrante quand on passe d'Aulnoy et ses contemporains à nos auteures. Les volumes 2 à 6 sont consacrés à la baronne, avec quelques extraits de Mailly, Auneuil et La Force. Ils comprennent quinze illustrations, et presque toutes font appel à l'imaginaire galant du salon par une intertextualité d'inspiration précieuse. Le collectionneur des années 1780-90, et le $\mathrm{XIX}{ }^{e}$ siècle après lui, disposent avec elles d'une topique résumée en quelques phrases, qui sort tout droit d'un roman ou d'une pastorale : «Je ne suis pas au Roi Madame, je suis à vous et je ne veux être qu'à vous", "Quelque respect que j'ai pour vous Madame permettez-moi de m'opposer au larcin que vous voulez me faire» reprennent une courtoisie usée. «Désaltérez-vous, beau Fanfarinet», «Voici pour le Pèlerin blessé», "Que faites-vous là, Bergère», "Le bruit qu'ils firent en marchant la tira de sa rêverie», «Jurez-vous une fidélité éternelle en présence de cette merveilleuse Fée?» ressuscitent une Astrée dont on ne lisait plus, souvent, que des adaptations. Si l'on passe les illustrations des 
contes orientaux, qui portent presque toujours des titres aux connotations attendues, on relève la présence de cinq conteuses dont les textes datent de 1722 au plus tôt. Parmi les dix extraits choisis, nombreux sont ceux qui invitent le lecteur à une nostalgie moins souriante : "Arrêtez, arrêtez barbares, inhumains», "Juste Ciel! C'est mon mari!», "Ménocrate, tu vas devoir ton bonheur à mes crimes», "Ses cris se font entendre jusques aux Cieux», "Traître, défends ta vie», "Ne craignez rien et daignez m'écouter un moment», ou, pour la princesse Baleine dans La Princesse Camion de $\mathrm{M}^{11 \mathrm{e}}$ de Lubert (1743), «Il faut m'épouser tout à l'heure ou m'écorcher toute vive». Chez les conteurs de la même veine, comme Saint-Hyacinthe ou Montcrif, on aura d'ailleurs de semblables exclamations. On n'est plus ici chez Scudéry ni Urfé, mais plutôt dans les palais sanglants d'un Crébillon père, ou dans les intrigues larmoyantes des années 1770. La théâtralisation se développe pour toutes les illustrations de roman, et les contes de fées n'en sont qu'un exemple parmi d'autres.

\section{Vision théâtrale et pédagogie}

Stéphane Lojkine ${ }^{31}$, à partir d'un poncif $\mathrm{du} \mathrm{XVIII}^{\mathrm{e}}$ siècle, le thème de Roger dans les bras d'Alcine, souvent confondu avec Renaud et Armide $^{32}$ ou n'importe quel couple mythologique ou romanesque Mars et Vénus, Vénus et Adonis - , montre comment l'image, de narrative au $\mathrm{XVI}^{\mathrm{e}}$ siècle, devient de plus en plus théâtrale. Ce qu'il met en évidence pour Fragonard ou Cochin relève de toute une évolution de la peinture. En ce deuxième dix-huitième siècle où la notion de tableau, dans les deux sens du terme, devient centrale, les illustrations de fiction sont avant tout des scènes. Elles évoluent d'ailleurs d'un contexte à l'autre : tapisseries, gravures, dessin. Mais elles véhiculent toujours, semble-t-il, des références à un modèle pictural, et donc, à ce moment, théâtral. Elles s'attachent souvent à un moment fort du récit, ou à l'instant qui précède la crise ${ }^{33}$.

31 «L'île d'Alcine, de l'allégorie à la chambre» (notice Utpictura 18).

32 Selon Lojkine, «la confusion avec Renaud et Armide s'explique encore par le fait que le Tasse, en imaginant l'épisode du chant XVI de la Jérusalem délivrée, s'est directement inspiré du chant VII du Roland furieux».

33 "Condensation" et «immobilisation", selon Christophe Martin, dans «Dangereux suppléments», op. cit., p. 31. Cette question a été abordée dans Aurélie Zygel-Basso, "Comme il est aisé de le voir dans l'estampe" : les contes merveilleux du "style 
Le spectaculaire intervient surtout dans les contes orientaux ou pseudo-grecs. Marillier utilise différents moyens picturaux pour le mettre en évidence. Il souligne le cadre de la scène représentée, recrée une ambiance qui plonge le lecteur et le spectateur dans l'effroi et sert de support au spectaculaire. Ainsi, dans la Première Veillée de Thessalie (Fig. 1), la pièce sombre uniquement éclairée par une lampe suspendue rend beaucoup plus extraordinaire l'apparition d'outre-tombe, entourée de fumée, du mari de Mélanie vêtu de longs voiles, tout en jouant son rôle technique de repoussoir. Marillier se sert également des jeux de lumière. Dans le Prince Désir (Fig. 3), il met en évidence la fée avec son nez minuscule, même si la rencontre entre celle-ci et le héros prête au comique. Cette «erreur de la nature» est redoublée avec les représentations des animaux : le cheval du Prince avec un long museau et celui du chien de la fée complètement écrasé. Le dessinateur s'appuie aussi sur une rhétorique du masque et de la gestuelle des personnages, comme dans la Première Veillée où Mélanie est effrayée par la vision de son mari. Elle a la bouche entrouverte et laisse deviner le cri d'effroi qui s'en échappe, les yeux écarquillés, et elle tend les mains en avant pour repousser la funeste apparition. Le dessinateur joue avec la lumière en éclairant la jeune fille. La présence de Micalé, peinte sous des traits repoussants (menton en galoche, nez crochu), ainsi que son geste de défense, rendent la scène encore plus saisissante. Marillier reprend très fréquemment les mêmes éléments. Dans la Troisième Veillée de Thessalie (Fig. 2), le lecteur assiste au suicide de Stéviane dont la méchanceté est découverte. Les costumes et décors de ces gravures théâtrales s'alignent sur une version simplifiée du «style à la grecque», suffisamment flou pour servir l'esthétique du tableau vivant.

Pour les contes féminins de 1740-1760, seule l'illustration du conte de $\mathrm{M}^{\mathrm{me}}$ Leprince de Beaumont, Le Prince Désir, est différente. Elle se rattache bien davantage au troubadour que Marillier avait affectionné pour les premiers volumes, notamment ceux consacrés aux œuvres de $\mathrm{M}^{\text {mes }} \mathrm{d}^{\prime}$ Aulnoy, d'Auneuil, $\mathrm{M}^{\mathrm{lle}}$ de La Force. Les contes du XVII ${ }^{\mathrm{e}}$ siècle finissant, textes d'autres Modernes, participent d'une relecture des «antiquités gauloises» bien connue. Ceux de $\mathrm{M}^{\mathrm{me}}$ Leprince de Beaumont veulent renouer le fil de cette tradition en durcissant la visée didactique, qui passe souvent chez elle au premier plan $^{34}$. Le compila-

nouveau" dans le Cabinet des fées de Mayer et Marillier", Actes du colloque international Jeunes chercheurs 2008 du CIERL, Université du Québec à Rimouski [sous presse, Cahiers du CIERL].

34 Sur le vraisemblable et le renouvellement du merveilleux, notamment chrétien, 
teur Mayer, y voit sans doute le signe d'une continuité poétique. Elle se traduit par un costume similaire, celui du passé Valois dont la référence idéalisée existait déjà dans les illustrations du XVII siècle, et qui relaie la vision pédagogique de la conteuse comme de l'éditeur.

Dans Le Prince Désir - conte pédagogique destiné aux jeunes enfants - Marillier met en lumière l'erreur dans laquelle est plongée le prince : la flatterie des courtisans l'empêche de voir ses propres défauts. Chacun se moque du nez de l'autre qui est l'opposé du sien. Le dessinateur rajoute dans la scène la servante qui apparaît plus tard dans le texte. Elle aussi flatte sa maîtresse sur son principal défaut : le bavardage. La servante sert alors de miroir aux courtisans du prince et l'illustration rejoint la vision de Leprince de Beaumont. Chez la conteuse, point d'esthétique du mensonge, mais une recherche de cohérence pour les petites élèves. On y apprend à distinguer le vrai du faux, les «histoires vraies» des contes. C'est toute une perspective de critique littéraire, subordonnée à l'éducation, qui se dessine. Le plaisir de la lecture n'est qu'un outil qui doit permettre d'affiner son jugement. En cela, Leprince de Beaumont, presque la seule femme dont la production de contes merveilleux soit significative après 1755 , propose une conception utilitariste du conte de fées qui s'intéresse moins aux questions de poétique qu'à une mise en scène de la lecture critique du merveilleux, dans un but pédagogique. Illustrer son Magasin des Enfants était difficile, il est d'ailleurs peu présent dans le Cabinet des fées.

Ainsi, les dessins que Marillier propose pour nos conteuses comme pour Duclos se tournent davantage vers une mode, sinon néoclassique, du moins à la grecque. Au contraire, les premiers auteurs, paradoxalement associés à Leprince de Beaumont, se voient illustrés dans une veine qui fait correspondre «Once upon a time» à «In the Middle Ages" ${ }^{35}$ " au sens très large du terme. Cependant, ces allusions picturales diverses sont à lire comme des facettes de la même esthétique rétrospective. Selon l'analyse de Daphne M. Hoogenboezem, dont les travaux permettent d'éclairer l'évolution du troubadour européen dans les illus-

dans les contes de Leprince de Beaumont, voir l'édition critique récemment parue d'Élisa Biancardi : Gabrielle-Suzanne Barbot, $\mathbf{M}^{\mathrm{me}}$ Jean-Baptiste de Gaallon de Villeneuve, La Jeune Américaine et les contes marins, Les Belles Solitaires, Marie Leprince de Beaumont, Le Magasin des enfants (Contes), É. Biancardi (éd.), Paris, H. Champion, coll. «Sources classiques», Bibliothèques des Génies et des Fées, 15, 2008. 
trations de contes ${ }^{36}$, la tendance apparaît dès les gravures bien connues de Clouzier pour l'édition princeps de Perrault par Barbin (1697, après le manuscrit de $1695^{37}$ ), et influence les illustrateurs tout au long du XVIII ${ }^{\mathrm{e}}$ siècle.

Par exemple, l'utilisation de la «voyeur perspective ${ }^{38}$ ", qui permet au spectateur de voir à la fois l'intérieur et l'extérieur du bâtiment grâce à un mur coupé, est très fréquente dans les illustrations de Mélusine de Jean d'Arras (1393) au XV siècle. On voit souvent Raimondin observer à travers un trou dans la porte de la pièce où la femme-serpent se baigne: «Illustrations [...] combining polyscenic picturing and the "voyeur perspective", are reused in almost all editions until the editions of the sixteenth and seventeenth centuries. One woodcut [...] appeared in the editions published in Paris by Jean Trepperel around 1527 and is reused by the editors Nicolas Oudot and Jacques Febvre in the Bibliothèque bleue in 1660, 1677 and $1692^{39}$ » (Fig. 5). Or, Marillier reprend cette veine d'illustration, en la tirant du côté du spectaculaire, dans la planche prévue pour La Princesse Camion de $\mathrm{M}^{\mathrm{lle}}$ de Lubert (1743, Fig. 4). Plus de mur mais une draperie qui révèle au Prince Zirphil, chasseur, la malédiction qui frappe la princesse Baleine, mi-femme, mi-poisson, à l'instar de Mélusine. La servante Citronette sert même d'auxiliaire au dessinateur pour montrer la scène, et sa main qui tient le rideau correspond au geste éloquent ${ }^{40} \mathrm{du}$ prince épouvanté, face à une baignoire décorée de frises, sur fond de colonnes. Mais comme le montre

36 Daphne M. Hoogenboezem, «Medievalism and Magic: Illustrating Classical French Fairy Tales», dans Wim van Anrooij, Alicia Montoya et Sophie van Romburgh (éds.), Early Modern Medievalisms, The Interplay between Scholarly Reflection and Artictic Production, Intersections, Yearbook for Early Modern Studies, 15, Leiden, 2010, 249-283, p. 266.

37 Gravures analysées, entre autres, par Tony Gheeraert, dans Perrault, Fénelon, Mailly, Préchac, Choisy et anonymes, Contes merveilleux, Tony Gheeraert (éd.), Paris, Honoré Champion, coll. "Sources classiques», BGF, 4, 2005. Voir aussi les gravures sur cuivre de Clouzier pour Les contes des fées de $\mathrm{M}^{\mathrm{me}} \mathrm{d}^{\prime}$ Aulnoy, chez le même Barbin. L'analyse de D. Hoogenboezem se base sur l'édition Meindert Uytwerf (La Haye, 1698) et La Compagnie des Libraires (Paris, 1725).

38 Daphne M. Hoogenboezem, op. cit. Voir L. Harf-Lancner, «L'Illustration du roman de Mélusine de Jean d'Arras dans les editions du XVe et du XVIe siècle», dans Le livre et l'image en France au XVIe siècle, Presses de l'Ecole Normale Supérieure, Paris, 1989, p. 29-55, et F. Clier-Colombani, La fée Mélusine au Moyen Age, Images Mythes et Symboles, Le Léopard d'or, Paris, 1991, p. 49.

39 Daphne M. Hoogenboezem, op. cit.

40 Sur la différence entre la parole ordinaire et la parole éloquente au XVII ${ }^{e}$ siècle, 
D. Hoogenboezem, Marillier n'est pas le premier à proposer au lecteur ce style syncrétique. Le graveur hollandais Simon Fokke (1712-1784), avec ou sans la collaboration du peintre français Jacques de Sève, illustre les contes d'Aulnoy publiés par Marc Michel Rey dans Le Cabinet des fées (Amsterdam, 1754-1773). Il mêle tour médiévale et colonnes d'un intérieur classique dans son illustration pour La Belle aux cheveux d'or. En Angleterre, les illustrations de contes d'Aulnoy par John Nicolson (1715) dépeignent avec précision cols de dentelle et corset, manteaux $\mathrm{d}$ 'hermine et bijoux à l'espagnole - une évocation, on l'a vu, du passé au sens large. Deux autres éditions anglaises intitulées Queen Mab (J. Dodsley, Londres, 1770 et Vernor \& Hood, Londres, 1799), pratiquent l'amalgame allusif par le biais de l'architecture et des costumes.

Dans tous les cas, la perspective qu'adopte la réception illustrée du deuxième $\mathrm{XVIII}{ }^{\mathrm{e}}$ siècle est double : presque toujours dramatique, et désormais largement patrimoniale. Elle donne à voir un costume semblable, celui du bon vieux temps dont la référence idéalisée existait déjà dans les illustrations du XVII siècle, et auquel le genre même du conte de fées, parce que révolu, appartient désormais tout entier. Les dessins de Marillier peignent donc aussi un Tableau des fées vivant, qui relève du drame : «dans l'esprit des années 1770, le ton de l'histoire ne répond pas au goût de l'éthéré mais à celui [...] du macabre, du noir [...]. Et [...] la vapeur noirâtre [...] ne sépare plus distinctement le réel de l'imaginaire mais menace plutôt le réel en s'y insinuant à partir d'un au-delà inconnaissable ${ }^{41}{ }^{\text {}}$. Marquée par le goût des antiquités «médiévales» au sens très large ${ }^{42}$, cette esthétique accompagne la valorisation du génie national. Mayer parlait des contes merveilleux comme de «tableaux de maître» dans son prospectus du Cabinet des fées. Mais le cabinet, à vrai dire, a vécu : le discours patrimonial fige le merveilleux lorsqu'il l'encadre pour l'accrocher dans son Musée.

\section{AURÉLIE ZYGEL-BASSO}

Université du Québec à Trois-Rivières

AMÉLIA BELIN

Université de Dijon

voir Marc Fumaroli, L'école du silence, le sentiment des images au XVIIe siècle, Paris, Flammarion, 1994, p. 324-342.

41 Philip Stewart, "Images de ce qui ne fut jamais», dans Le Conte merveilleux au XVIII siècle. Une poétique expérimentale, éd. R. Jomand-Baudry et J.-F. Perrin, Paris, Kimé, 2002, p. 376.

42 En France, mais aussi aux Pays-Bas et en Angleterre. 
86 Aurélie Zygel-Basso et Amélia Belin

\section{Illustrations}
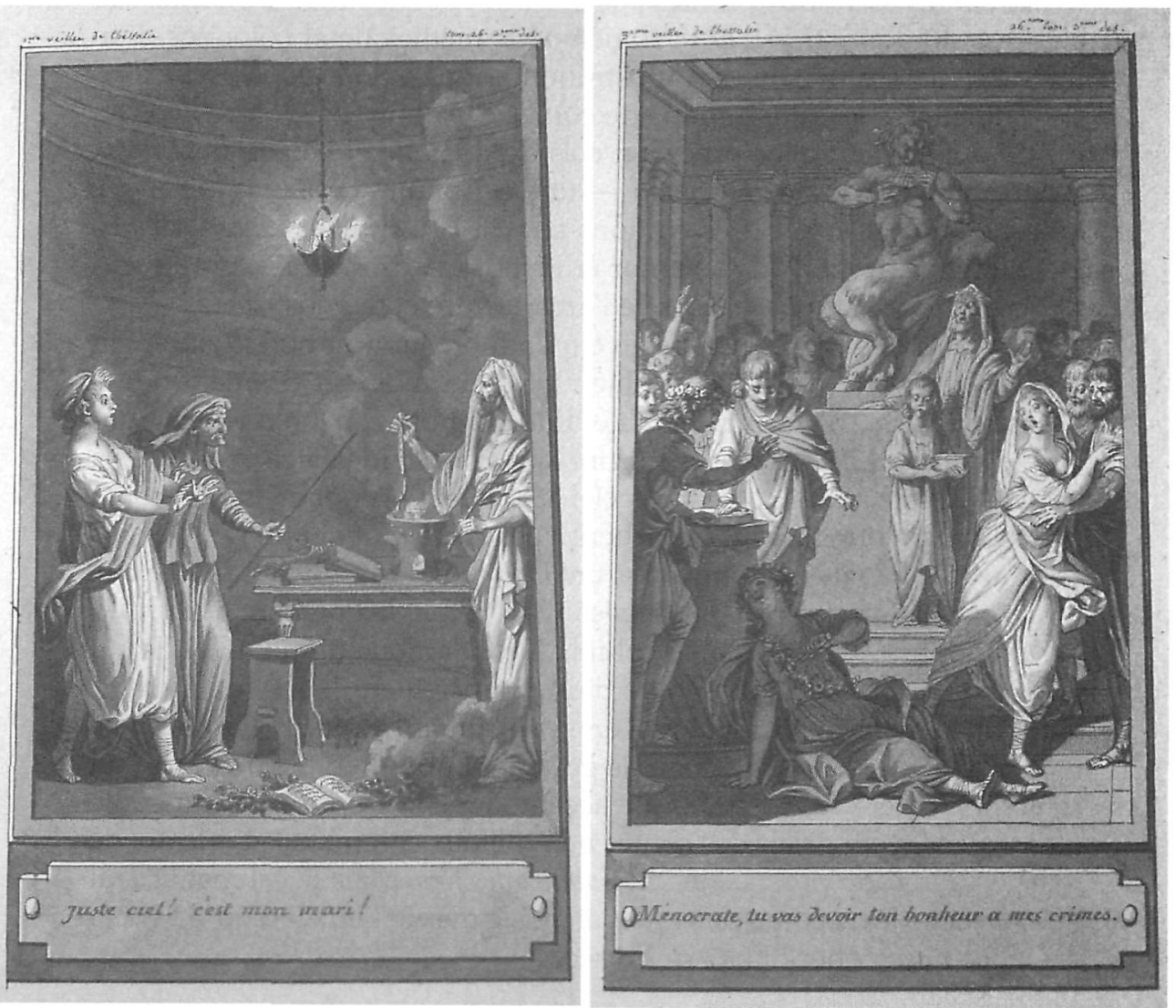

$M^{\text {lle }}$ de Lussan, Les Veillées de Thessalie (1731-1741), veillées I et III Dessins préparatoires du Cabinet des Fées par C.-P. Marillier (v. 1785) (BnF, Dptt des Mss occidentaux, Fonds Rothschild IV. 1. 11-12, T. $2 n^{\circ} 79$ et $n^{\circ} 80$ ). 


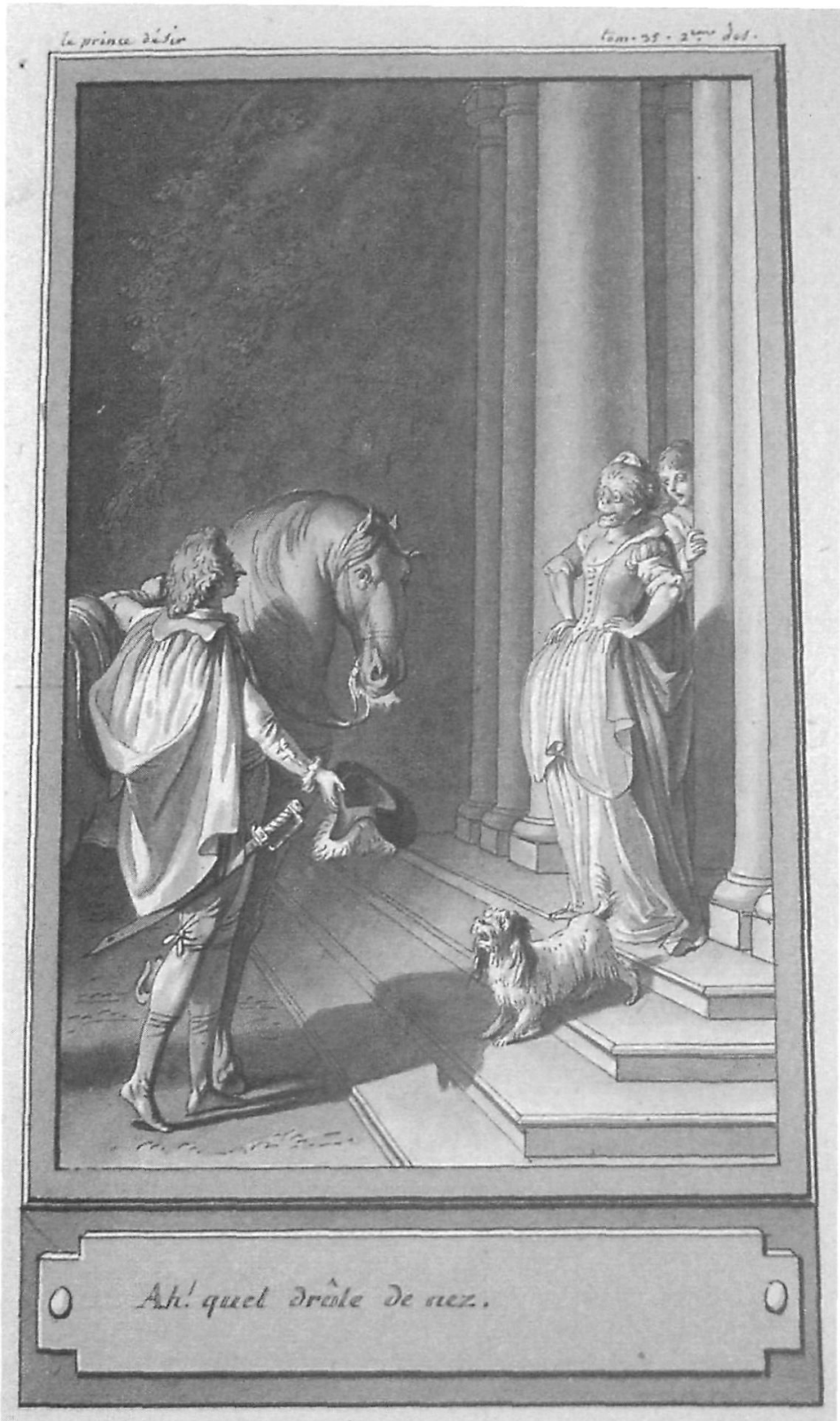

$\mathrm{M}^{\mathrm{me}}$ Leprince de Beaumont, Le Prince Désir et la Princesse Mignonne (Le Magasin des enfants, 1757)

Dessin préparatoire du Cabinet des Fées par C.-P. Marillier (v. 1785) (BnF, Dptt des Mss occidentaux, Fonds Rothschild, IV. 1. 11-12, T. 2 n¹06). 


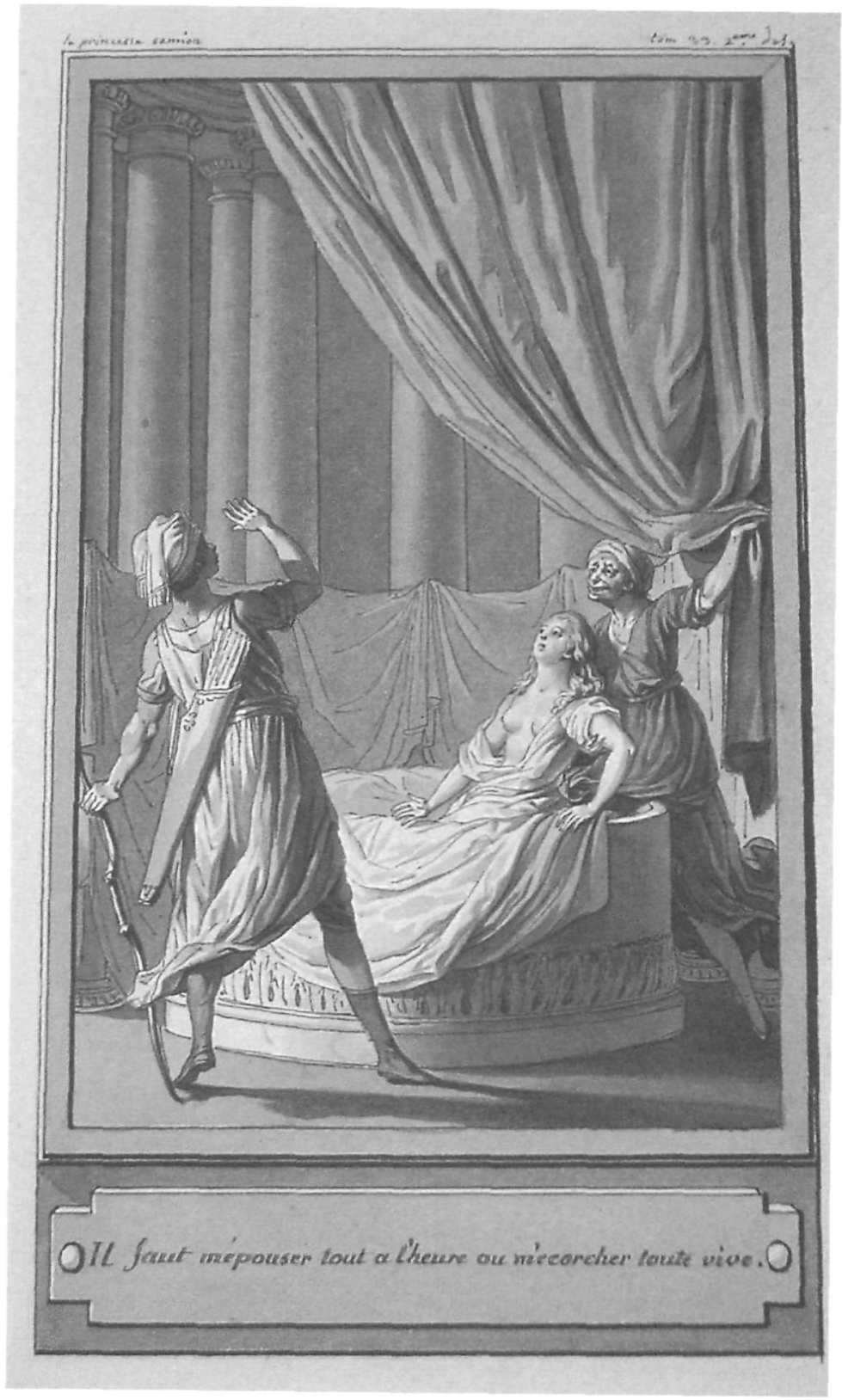

$\mathrm{M}^{\mathrm{Ile}}$ de Lubert, La Princesse Camion (1743)

Dessin préparatoire du Cabinet des Fées par C.-P. Marillier (v. 1785)

Fonds Rothschild, IV. 1. 11-12 (BnF, Dptt des Mss occidentaux), T. 2 n¹00. 


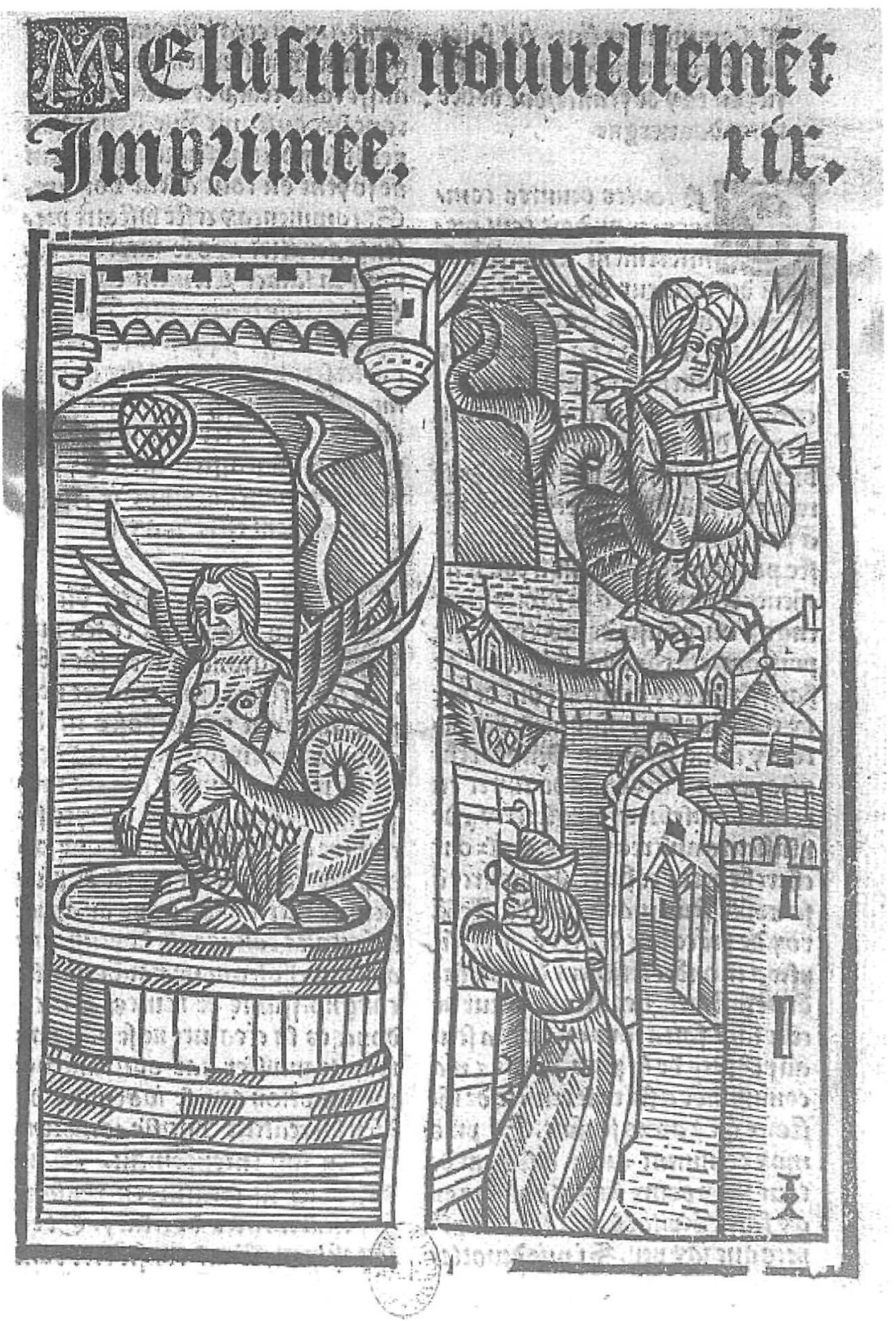

Anonyme, gravure sur bois pour Jean d'Arras, Mélusine, Nouvellement imprimée, Jean Trepperel, Paris, 1527-1532 (BnF, Rés. Y22788). 